\title{
"Self-Heating" Enabled One-Pot Synthesis of Fluorescent Carbon Dots
}

\author{
Xiaoyu Ma, ${ }^{1 *}$ Wei Zhong, ${ }^{2}$ Jing Zhao, ${ }^{3}$ Steve L. Suib ${ }^{2,3}$ and Yu Lei ${ }^{1,4^{*}}$
}

Instead of using traditional external heating treatment methods, a facile heating equipment-free method is developed for one-pot synthesis of fluorescent carbon dots (CDs) via exothermic crosslinking reaction enabled "self-heating" and further induced high degree of carbonization. The obtained CDs exhibit strong blue fluorescence and are well characterized through a series of advanced analysis, including their optical properties, surface morphology, and chemical compositions. The corresponding synthesis mechanism is also disscussed and proposed. Moreover, the assynthesized carbon dots have been successfully applied for selective $\mathrm{Fe}^{3+}$ detection as a simple demonstration. $\mathrm{Fe}^{3+}$ can significantly quench the fluorescence signal of CDs, whereas other metal ions show insignificant effect on their fluorescence intensity. The quenching performance is ascribed to synergistic effect of electrostatic interaction between ferric ions and the fluorescent CDs as well as interaction induced electron transfer. This study expands the cutting edge of simple synthesis of fluorescent CDs, where CDs hold great promises in sensing applications.

Keywords: Carbon dots; One-pot; Fluorescence; Self-heating; Fe ${ }^{3+}$ sensing

Received 3 April 2019, Accepted 8 July 2019

DOI: $10.30919 / \mathrm{es} 8 \mathrm{~d} 805$

\section{Introduction}

Carbon dots (CDs), discovered as a new type of fluorescent nanomaterial in 2004, have attracted more and more attention in recent years, due to their advanced optical properties, good biocompatibility, chemical stability, and easiness of synthesis. They have been applied in a wide span of applications, including bio-sensing, bio-imaging, drug delivery, medical diagnosis, and catalysis, etc.. ${ }^{1}$ The optical properties of $\mathrm{CDs}^{2-6}$ are employed as a determining factor for potential applications of CDs. Consequently, it has become a major research subject to develop novel methods to synthesize CDs with desired optical properties in recent years. Since the first report about the synthesis of CDs, condensation and chemical doping have been extensively employed in synthesis to obtain CDs with different fluorescence properties. A number of publications have suggested that doping of heteroatoms such as nitrogen, sulfur, and phosphorus can alter the optical properties of the CDs. ${ }^{7-9}$ Moreover, surface chemical groups of CDs are also believed to be an important factor on their luminescent properties, which are generally comprised of $\mathrm{sp}^{2} / \mathrm{sp}^{3}$ carbon and oxygen/nitrogen-containing chemical groups. ${ }^{10,11}$ Although the exact mechanism of CD fluorescence is still controversial, three theories have been proposed, including quantum confinement effects, surface states, and molecular states. ${ }^{10}$

Over the past decade, a series of studies have employed various precursors and synthetic methods for the fabrication of CDs. They can be sorted into two approaches: top-down and bottom-up methods. In the top-down route, the carbon nanoparticles are fabricated via breaking large pieces of materials into nanostructured materials by various reactions, such as laser ablation, ${ }^{12}$ electrochemical oxidation, ${ }^{13}$ arc charge, ${ }^{1}$ etc.. In the bottom-up synthesis strategy, CDs are synthesized involving a sequence of steps to assemble the precursors through a thermal treatment, ${ }^{14}$ a microwave-assisted method, ${ }^{15}$ and the use of supports, ${ }^{16}$ where heat and/or pressure induced agglomeration drives the formation of fluorescent CDs.

\footnotetext{
${ }^{1}$ Department of Biomedical Engineering, University of Connecticut, 06269, USA

${ }^{2}$ Institute of Materials Science, University of Connecticut, 06269, USA

${ }^{3}$ Department of Chemistry, University of Connecticut, 06269, USA

${ }^{4}$ Department of Chemical and Biomolecular Engineering, University of Connecticut, 06269, USA

*E-mail:yu.lei@uconn.edu (Y.Lei ); xiaoyu.ma@uconn.edu (X. Y. Ma)
} 
Using bottom-up approaches, the doping of nitrogen atoms in CDs is widely investigated, due to the ability to adjust photoluminescence properties, surface features, and the electrochemical behaviors of the synthesized CDs. ${ }^{17,18}$ For example, citric acid doped with different kinds of amines, serves as an environment-friendly carbon source in the formation of CDs, ${ }^{19-21}$ by a one-step pyrolysis treatment. However, elevated temperature treatment or microwaveassisted strategies were typically required to assist the carbonization, where heating equipment is always a prerequisite.

In this study, we report a heating equipment-free strategy to synthesize CDs, where the schematic synthesis process is shown in Fig. 1. In this approach, the high extent of carbon condensation process is accomplished in two parallel routes in one pot: initial crosslinking conjugation between carbon source and cross-linker enables "self-heating", which is attributed to the exothermic cross-linking reaction; and "self-heating" further induces carbonization, forming fluorescent CDs. As a demonstration in this study, citric acid, comprising of three reactive carboxyl groups, was selected as a model compound serving as the major carbon source. Meanwhile, 1-ethyl-3-(3dimethylaminopropyl) carbodiimide hydrochloride (EDC) serves as a model cross-linker and nitrogen doping source. The as-synthesized CDs, displaying strong blue fluorescence under UV lamp, exhibit excitation and $\mathrm{pH}$ independent emission properties. Furthermore, as a demonstration, the fluorescent CDs have been employed as chemical sensor for selective $\mathrm{Fe}^{3+}$ detection, and the sensing mechanism has been systematically investigated, where fluorescence quenching of CDs upon addition of $\mathrm{Fe}^{3+}$ is ascribed to electrostatic interaction as well as electron transfer. Compared to traditional external heating treatment induced carbonization, the equipment-free method expandes a novel synthesis method of carbon nanoparticles.

\section{Experimental}

\subsection{Reagent and Chemicals}

Citric acid (anhydrous 99.5\%) and 1-ethyl-3- (3dimethylaminopropyl) carbodiimide hydrochloride (EDC) were purchased from Fisher Scientific. Metal ion solutions were prepared using a series of chemicals, such as $\mathrm{FeCl}_{3}$, $\mathrm{CuCl}_{2}, \mathrm{KCl}$, etc. Different $\mathrm{pH}$ buffer solutions were prepared using various chemicals, including sodium citrate, hydrochloric acid, sodium phosphate dibasic, sodium phosphate monobasic, sodium hydroxide, and sodium tetraborate.

\subsection{Synthesis of Fluorescent Carbon Nanoparticles}

As shown in Fig. 1, $0.4 \mathrm{M}$ citric acid (CA) aqueous solution was mixed with the same volume of $2 \mathrm{M} \mathrm{EDC}$ for carbonization, where exothermic crosslinking reaction serves as the intrinsic thermal generating source. The CDs solution was allowed to sit overnight for stabilization and then used for the following characterization and sensing applications without further purification.

\subsection{Characterization}

Prior to optical properties analysis, CDs were diluted 100 times in DI water. The absorbance spectra were collected using a Cary 50 UV-vis spectrophotometer (Agilent Technologies). Varian Cary Eclipse fluorescence spectrometer (Agilent Technologies) was employed to obtain fluorescence emission spectra. The electrospray ionization mass spectrometry (ESIMS) spectra were conducted using AB Sciex Ostar Elite spectrometer. CDs solution was diluted in a water/methanol (50:50 v/v) mixture with an infusion rate at $10 \mu \mathrm{L} \mathrm{min}$. Fourier transform infrared (FTIR) spectra (Fig. 2d) of the CDs was collected using a Nicolet 560 Fourier spectrometer with a Diamond ATR accessory. A quantum 2000 Scanning ESCA Microscope with a monochromatic A1 K $\alpha$ X-ray source, was applied to collect X-ray photoelectron spectra (XPS) spectra of the CDs.

\subsection{Detection of Metal Ion in the Aqueous Phase}

The sensing application of the CDs was conducted through the addition of target metal ion solution into CDs, where the fluorescence quenching performance was monitored by the fluorescence spectrophotometer with excitation/emission slit widths at $5 \mathrm{~nm}$ after mixing. Briefly, The CDs solution was diluted 60 times and placed in a $3 \mathrm{~mL}$ disposal cuvette, where the target metal ion analyte solutions were titrated

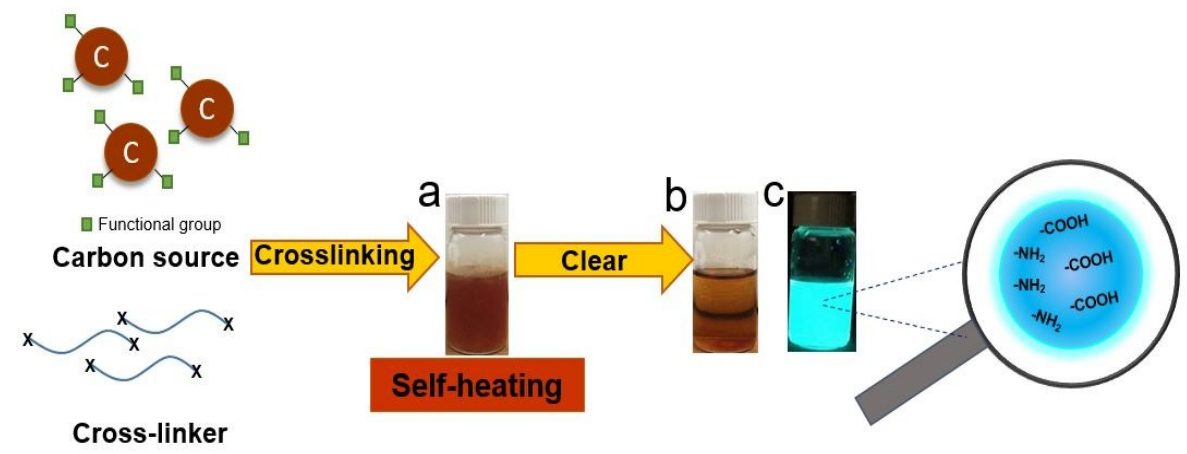

Fig. 1 Scheme of the one-pot synthetic strategy for carbon dots. 
with at least three titrations. The fluorescence emission was recorded in the wavelength range of $400 \sim 600 \mathrm{~nm}$ with the excitation wavelength at $390 \mathrm{~nm}$. To characterize the quenching behavior, the Stern-Volmer plot has been employed to further investigate sensing mechanism.

\section{Results and Discussion}

\subsection{Synthesis of the CDs and their Optical Properties}

The synthesis process of CDs is completed through two parallel mechanisms: carbon source is initially condensed and intermolecularly dehydrated to form oligomers via crosslinking reaction; meanwhile, the exothermic crosslinking reaction enables the increasing of temperature in the solution, which leads to the further carbonization through a burst of a high concentration of nucleation. The synthesis approach is briefly illustrated in Fig. 1. Firstly, EDC crosslinking CA results in a cloudy solution due to the formation of insoluble ester bonds between them, as shown in the Fig. 1a. Simultaneously, this reaction produces a large amount of heat, serving as an intrinsic hydrothermal treatment process, which induces the formation of the CDs. This process is also presumably accompanied by the gradual decomposition of ester bonds under acidic condition. Finally, a dark brown solution of CDs was obtained (Fig. 1b), which was used for subsequent experiments without purification. As an observation, the solution became cloudy immediately after mixing of the two precursors. After several minutes, the solution gradually turned into a clear dispersion of CDs (Fig. 1b). According to Fig. 1c, the obtained CDs solution exhibits strong blue-green fluorescence under $365 \mathrm{~nm}$ UV light.

\subsection{Optical Properties of the CDs}

In the UV-vis spectrum of the CDs (Fig. 2a), there are two obvious broad absorption features in the range of $350 \mathrm{~nm} \sim 390$ $\mathrm{nm}$ and $450 \mathrm{~nm} \sim 500 \mathrm{~nm}$, respectively. The appearance of these two broad absorbance humps indicates the synergistic effects between the surface/molecule state and carbon core. These two peaks suggest an aromatic system, where a $\pi-\pi^{*}$ transition is ascribed to $\mathrm{C}=\mathrm{C}$ bonds, and $\mathrm{n}-\pi^{*}$ transitions is attributed to $\mathrm{C}$ $=\mathrm{O}$ and $\mathrm{C}=\mathrm{N}$ bonds, respectively. ${ }^{22}$ The bright blue-green fluorescence and highly clear CDs solution can be observed under UV light and visible light as shown in the Fig. $1 \mathrm{~b}$ and c. The fluorescence property is then investigated through emission scans at different excitation wavelengths in the range of $350 \mathrm{~nm}$ to $450 \mathrm{~nm}$ with $10 \mathrm{~nm}$ intervals (Fig. 2b). No obvious shift of the emission spectra is observed when changing the excitation wavelengths, which is attributed to the low number of surface defects of the synthesized CDs. ${ }^{23}$ Furthermore, it has been noted that it exhibits low emission spectra as excitation wavelength above $430 \mathrm{~nm}$. Although there is an obvious absorption feature in the range of $450 \mathrm{~nm} \sim 500$ $\mathrm{nm}$, no fluorescence is observed when the excitation wavelength is longer than $450 \mathrm{~nm}$ according to Fig. $2 \mathrm{~b}$. Therefore, the results indicate the formation of one kind of emitted species during synthesis process. ${ }^{24}$

The stability of as-synthesized CDs in different $\mathrm{pH}$
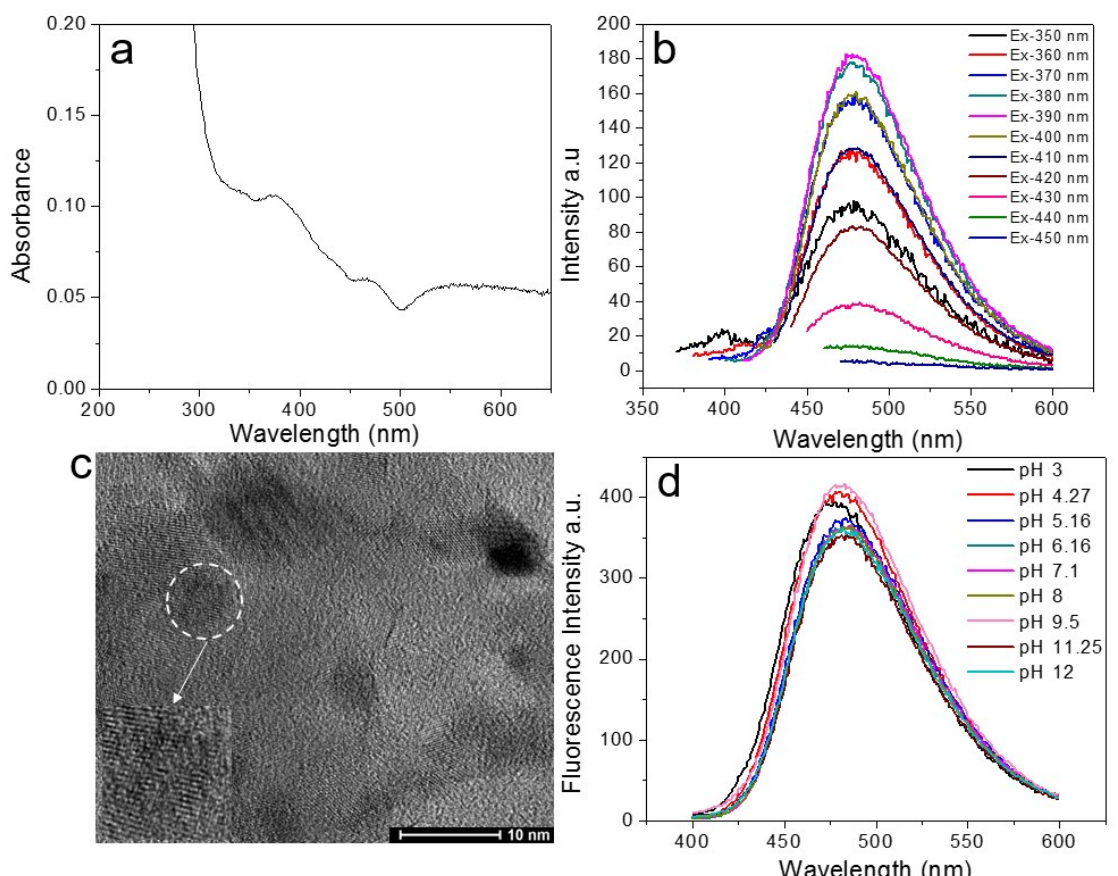

Fig. 2 (a) UV-vis spectra of as-synthesized CDs. (b) Fluorescence emission scans of CDs with different excitation wavelengths. (c) High resolution TEM image of the CDs. (d) Fluorescence emission spectra of CDs diluted in buffers with different $\mathrm{pH}$ values. 
levels is evaluated by recoding emission spectra of CDs diluted in buffers with various $\mathrm{pH}$ values, and the results are displayed in Fig. 2d. Within either extreme acidic condition $(\mathrm{pH} 3$ and $\mathrm{pH} 4.27$ ) or basic environment ( $\mathrm{pH} 9.5$ ), they exhibit slightly higher peak value of the fluorescent emission spectra, while besides these $\mathrm{pH}$ buffer conditions, fluorescence emission spectra of CDs are almost unchanged at all other $\mathrm{pH}$ levels. Overall, the as-prepared CDs show the $\mathrm{pH}$-independency property, and thus may serve as a robust fluorescent materials for various potential applications, such as optical based sensing, imaging, etc..

\subsection{Morphology and Composition of CDs}

ESI-MS spectrum was performed to analyze the composition of the as-prepared CDs and the corresponding result is presented in Fig. S1. The result suggests a mixture of the final solution, where the peaks represent CDs, citric acid, EDC and low-molecular weight oligomer-like compounds. According to the HRTEM image (Fig. 2c), some of the CDs are amorphous, while some exhibit wellresolved lattice fringes, which is attributed to highly disordered carbon atoms. Therefore, it indicates a mixed composition in the CDs solution, which is in good agreement with ESI-MS result.

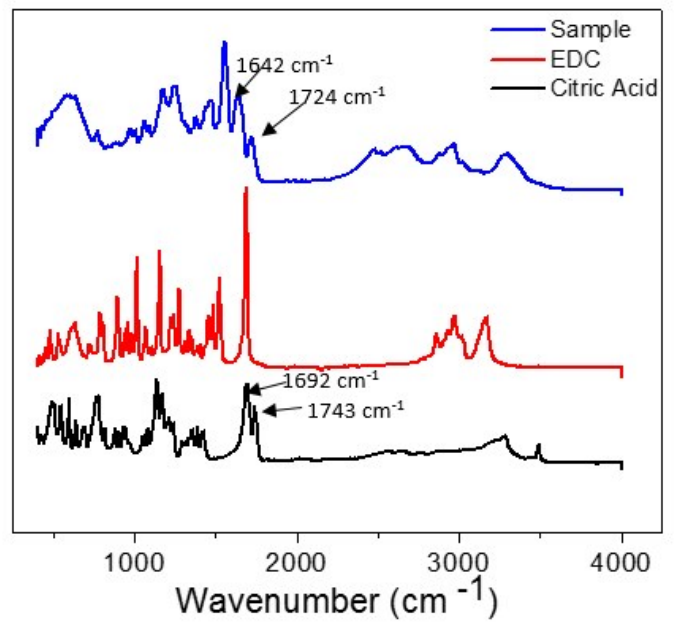

Fig. 3 FTIR spectra of CDs, citric acid and EDC, respectively.

To analyze the composition and functional groups, FTIR spectroscopy was employed to collect the IR spectra of citric acid and EDC with the corresponding results (Fig. 3). The two peaks at $1692 \mathrm{~cm}^{-1}$ and $1743 \mathrm{~cm}^{-1}$ in the FTIR spectra of citric acid arise from the $\mathrm{C}=\mathrm{O}$ stretch in the carboxylic groups. As shown in the spectra, these two peaks slightly shifted to lower wavenumbers in the FTIR spectra of CDs, which is ascribed to the conjugation reaction and the formation of - $\mathrm{N}-\mathrm{H}$ groups, indicative of the formation of CDs. ${ }^{22}$ Moreover, the FTIR spectrum of CDs shows the C-NH-C absorption peak at 1400 $\mathrm{cm}^{-1} .^{22}$ In addition, several IR absorption peaks are present in the FTIR spectrum of CDs such as $1249 \mathrm{~cm}^{-1}, 1558 \mathrm{~cm}^{-1}$, and $3290 \mathrm{~cm}^{-1}$, corresponding to $\mathrm{C}-\mathrm{N}$ of aromatic amines, $\mathrm{C}=\mathrm{N}$ stretching, and $\mathrm{N}-\mathrm{H}$ in combination with $\mathrm{O}-\mathrm{H}$ absorptions, respectively. The presence of these groups on CDs endows good hydrophilicity, which is indirectly corroborated by the good stability of CDs in aqueous solution.

Moreover, surface components and functional groups were resolved through X-ray photoelectron spectra (XPS) analysis. The XPS survey spectrum for the CDs is presented in Fig. 4a, suggesting that carbon, nitrogen, and oxygen are the major components. The high resolution spectrum of $\mathrm{C} 1 \mathrm{~s}$ exhibits four main binding energy peaks (Fig. 4b). They are $283.9 \mathrm{eV}, 284.8 \mathrm{eV}, 285.5 \mathrm{eV}$, and $287.3 \mathrm{eV}$, attributed to $\mathrm{sp}^{2}$ $\mathrm{C}=\mathrm{C}, \mathrm{C}-\mathrm{C}, \mathrm{C}=\mathrm{N}$ and $\mathrm{O}-\mathrm{C}=\mathrm{O}$, respectively. The $\mathrm{N} 1 \mathrm{~s}$ spectrum (Fig. 4c) indicates the presence of $\mathrm{C}=\mathrm{N}$ and $\mathrm{N}-\mathrm{H}$ groups, demonstrating that the as-prepared $\mathrm{CDs}$ are nitrogen-doped carbon nanoparticles. Moreover, the $\mathrm{O} 1 \mathrm{~s}$ spectrum (Fig. 4d) can be divided into three major peaks of different binding energy, revealing the presence of $\mathrm{C}=\mathrm{O}$, O$\mathrm{H}$, and $\mathrm{C}-\mathrm{O}$. The XPS results are in good agreement with FTIR results and also corroborate the observation of good solubility and strong blue-green fluorescence (nitrogen doping) of the as-prepared CDs.

\subsection{Detection of Metal Ion in the Aqueous Solution}

CDs have been applied for chemical detection and biosensing due to their stable optical properties and water solubility. ${ }^{11,25}$ In our study, we attempted to detect $\mathrm{Fe}^{3+}$ ions using the assynthesized CDs. $\mathrm{Fe}^{3+}$ ion is an important species presented in both clinical and environmental samples. ${ }^{26}$ It is particularly important to monitor the concentration of $\mathrm{Fe}^{3+}$ ion in biological system as it involves in a number of metabolic processes. ${ }^{27}$ For $\mathrm{Fe}^{3+}$ detection, the fluorescence of CDs was monitored when different amount of $\mathrm{Fe}^{3+}$ was added to the CDs solution. According to Fig. 5a, as the incremental addition of $\mathrm{Fe}^{3+}$ from $20 \mu \mathrm{M}$ to $900 \mu \mathrm{M}$ into the 60 -fold diluted CDs aqueous solution, the fluorescence of CDs was gradually quenched. The sensing capability of $\mathrm{CDs}$ to $\mathrm{Fe}^{3+}$ can be attributed to two effects. On one hand, the surface charge of the CDs may change when $\mathrm{Fe}^{3+}$ ions bind to them, decreasing their fluorescence; on the other hand, the effective coordination or chelation between $\mathrm{Fe}^{3+}$ and various - $\mathrm{OH},-\mathrm{COOH}$, and $-\mathrm{NH}$ groups on the surface of $\mathrm{CDs}^{28}$ results in electron-transfer induced fluorescence quenching. Moreover, the Stern-Volmer equation ${ }^{29}$ was employed to investigate the quenching behavior of CDs upon the addition of $\mathrm{Fe}^{3+}$. The linear fitting of the SternVolmer plot shows a high linear correlation $\left(\mathrm{R}^{2}=0.996\right)$, as shown in Fig. 5b, suggesting the good static quenching mechanism of carbon dots upon titration of $\mathrm{Fe}^{3+}$. In order to fit 
linear S-V curve, a linear quenching equation has been applied:

$$
\frac{I_{0}}{I}=K_{s v}[A]+1
$$

where, $I_{0}$ is the initial fluorescence intensity of CDs with no addition of target analyte, $I$ is the fluorescence intensity upon addition of certain amount of analytes, $K_{s v}$ is the quenching constant $\mathrm{M}^{-1}$, and $[A]$ is the molar concentration of the analyte. The quenching constant has been calculated according to the equation 1 , where $K_{s v}=1 \times 10^{3} \mathrm{M}^{-1}$. The large value of $K_{s v}$ demonstrates a high sensitivity of CDs to $\mathrm{Fe}^{3+}$, which might be attributed to the lower LUMO energy of the CDs once complexed with $\mathrm{Fe}^{3+}$.

In addition, the selectivity of the fluorescent CDs toward $\mathrm{Fe}^{3+}$ was also examined by titrating $\mathrm{Fe}^{3+}$ and other metal ions $\left(\mathrm{Cu}^{2+}, \mathrm{Co}^{2+}, \mathrm{Pb}^{2+}, \mathrm{K}^{+}\right.$, and $\left.\mathrm{Ca}^{2+}\right)$ into 60 -fold diluted $\mathrm{CDs}$ aqueous solution with a final concentration of 1 $\mathrm{mM}$. The results are shown in Fig. S2. The fluorescence intensity of CDs with $1 \mathrm{mM} \mathrm{Fe}^{3+}$ is quenched by $\sim 60 \%$, while all other metal ions only show insignificant quenching of the fluorescence intensity of CDs, indicating the good selectivity of the as-synthesized CDs toward $\mathrm{Fe}^{3+}$ detection.
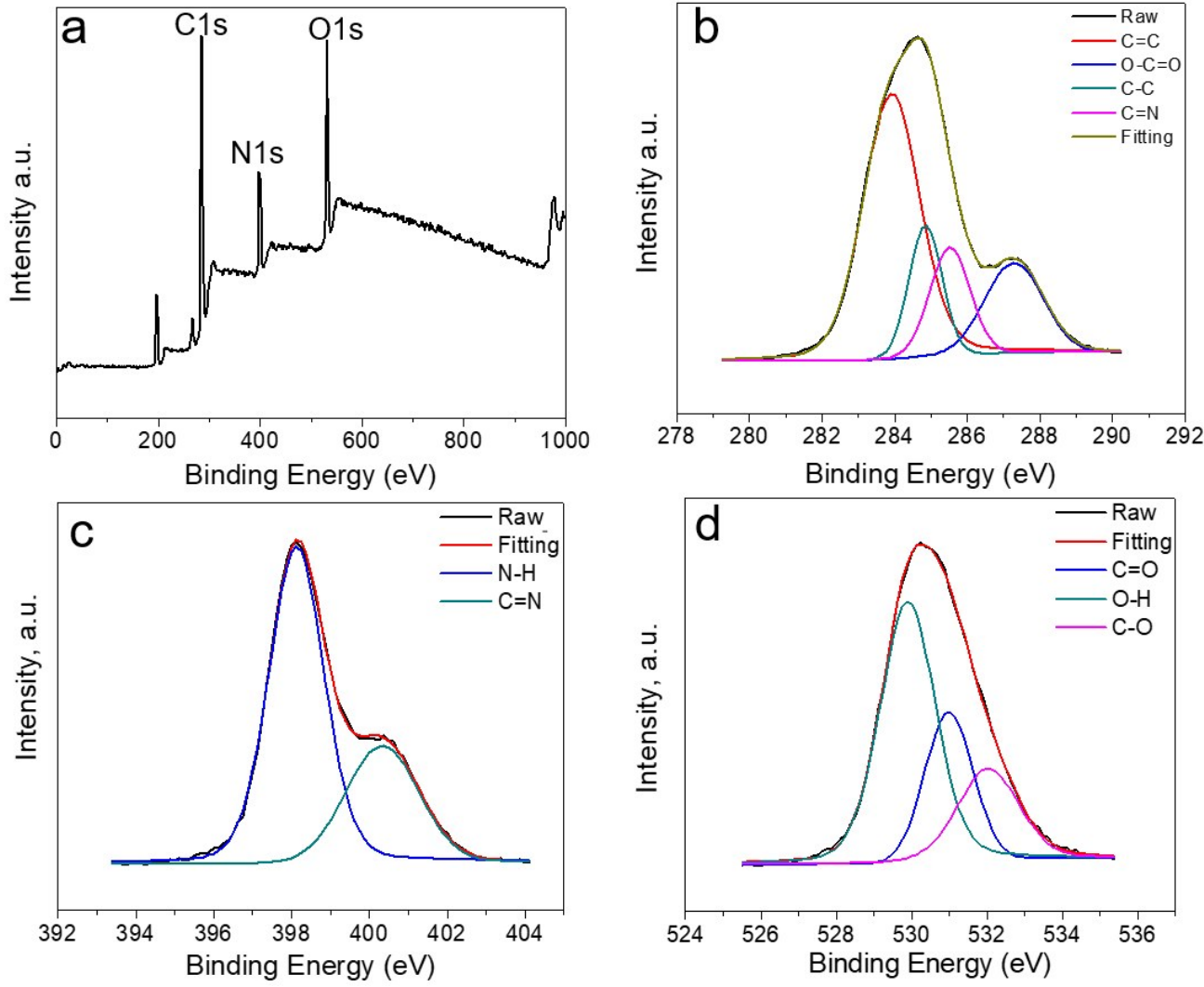

Fig. 4 XPS spectra of the CDs: (a) XPS survey spectrum. (b) High resolution of C 1s. (c) High resolution of $\mathrm{N} 1 \mathrm{~s}$. (d) High resolution of $\mathrm{O} 1 \mathrm{~s}$.
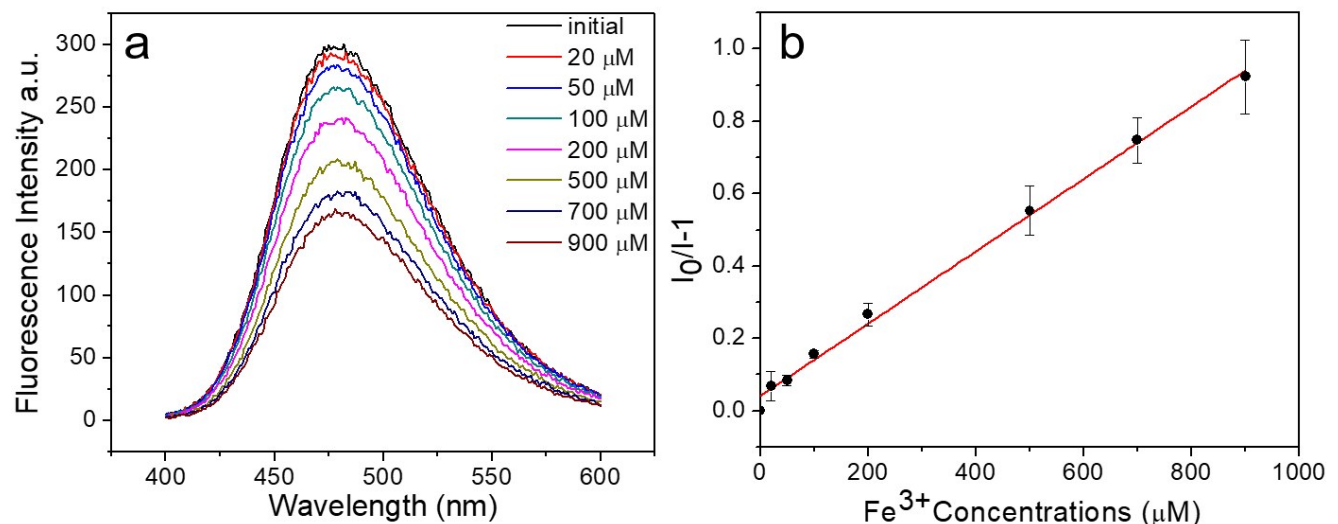

Fig. 5 (a) Fluorescence spectra of the CDs with the titration of $\mathrm{Fe}^{3+}$ in aqueous solution. (b) The Stern-Volmer plot for $\mathrm{Fe}^{3+}$ (The red line is the fitting curve). 


\section{Conclusions}

In summary, we have successfully developed a facile heating equipment-free method for one-pot synthesis of fluorescent carbon dots (CDs) via exothermic crosslinking reaction enabled "self-heating" and further induced high-degree of carbonization. Herein, citric acid and chemical crosslinker EDC were used as the model compounds to induce chemical crosslinking. The as-synthesized CDs exhibit a strong bluegreen fluorescence spectrum whose peak position is independent to excitation wavelength, suggesting only one emission species formed in the reaction and whose peak intensity is insignificantly dependent on the $\mathrm{pH}$ levels of the aqueous solution. The morphology and surface composition of the as-prepared CDs solution were characterized with multiple advanced techniques including TEM, FTIR, and XPS. As a demonstration, the as-prepared CDs were successfully applied for highly selective $\mathrm{Fe}^{3+}$ detection in aqueous solution over a number of other metal ions, such as copper, calcium, potassium, etc.. The Stern-Volmer equation was applied to investigate the sensing performance, where a linear fitting curve was plotted with a quenching constant of $1 \times 10^{3} \mathrm{M}^{-1}$, suggesting a static quenching mechanism. The excellent quenching performance is ascribed to charge effect as the chelation between $\mathrm{Fe}^{3+}$ and CDs. The present study provides a simple, low cost, and external thermal treatment free method for the synthesis of fluorescent CDs. Due to the simplicity, the unique fluorescent properties and aqueous stability of the assynthesized CDs, such material holds great promise in a wide range of applications beyond sensing.

\section{Conflict of interest}

There are no conflicts to declare.

\section{Acknowledgements}

This work was performed in part at the Biosciences Electron Microscopy Facility of the University of Connecticut. X. Y. M Thanks the support from UConn Dissertation Fellowship.

\section{References}

1. X. Sun and Y. Lei, TrAC-Trends. Anal. Chem., 2017, 89, 163-180.

2. Y. Fan, H. Cheng, C. Zhou, X. Xie, Y. Liu, L. Dai, J. Zhang and L. Qu, Nanoscale, 2012, 4, 1776-1781.

3. K. Jiang, S. Sun, L. Zhang, Y. Lu, A. Wu, C. Cai and H. Lin, Angew. Chem. Int. Edi., 2015, 54, 5360-5363.
4. S. Khan, A. Gupta, N. C. Verma and C. K. Nandi, Nano lett., 2015, 15, 8300-8305.

5. A. Sharma, T. Gadly, A. Gupta, A. Ballal, S. K. Ghosh and M. Kumbhakar, J. Phys. Chem. Lett., 2016, 7, 3695-3702.

6. A. Sciortino, E. Marino, B. V. Dam, P. Schall, M. Cannas and F. Messina, J. Phys. Chem. Lett., 2016, 7, 3419-3423.

7. S. N. Baker and G. A. Baker, Angew. Chem. Int. Edi., 2010, 49, 67266744 .

8. Y. Dong, H. Pang, H. B. Yang, C. Guo, J. Shao, Y. Chi, C. M. Li and T. Yu, Angew. Chem. Int. Edi., 2013, 52, 7800-7804.

9. S. Hu, A. Trinchi, P. Atkin and I. Cole, Angew. Chem. Int. Edi., 2015, 54, 2970-2974.

10. S. Zhu, Y. Song, X. Zhao, J. Shao, J. Zhang and B. Yang, Nano Res., 2015, 8, 355-381.

11. S. Y. Lim, W. Shen and Z. Gao, Chem. Soc. Rev., 2015, 44, 362-381.

12. Y. P. Sun, B. Zhou, Y. Lin, W. Wang, K. S. Fernando, P. Pathak, M. J. Meziani, B. A. Harruff, X. Wang and H. Wang, J. Am. Chem. Soc, 2006, 128, 7756-7757.

13. J. Zhou, C. Booker, R. Li, X. Zhou, T. K. Sham, X. Sun and Z. Ding, J. Am. Chem. Soc., 2007, 129, 744-745.

14. H. Liu, T. Ye and C. Mao, Angew. Chem. Int. Edi. 2007, 46, 6473-6475.

15. S. Qu, X. Wang, Q. Lu, X. Liu and L. Wang, Angew. Chem., 2012, 124, 12381-12384

16. R. Liu, D. Wu, S. Liu, K. Koynov, W. Knoll and Q. Li, Angew. Chem., 2009, 121, 4668-4671.

17. B. Shi, L. Zhang, C. Lan, J. Zhao, Y. Su and S. Zhao, Talanta, 2015, 142, 131-139.

18. S. Liu, R. Liu, X. Xing, C. Yang, Y. Xu and D. Wu, RSC Adv., 2016, 6 , 31884-31888.

19. M. J. Krysmann, A. Kelarakis, P. Dallas and E. P. Giannelis, J. Am. Chem. Soci., 2011, 134, 747-750.

20. X. Li, S. Zhang, S. A. Kulinich, Y. Liu and H. Zeng, Sci. Rep., 2014, 4.

21. X. Zhai, P. Zhang, C. Liu, T. Bai, W. Li, L. Dai and W. Liu, Chem. Commun., 2012, 48, 7955-7957.

22. M. Zhou, Z. Zhou, A. Gong, Y. Zhang and Q. Li, Talanta, 2015, 143, 107-113.

23. X. Sun, J. He, Y. Meng, L. Zhang, S. Zhang, X. Ma, S. Dey, J. Zhao and Y. Lei, J. Mater. Chem. A, 2016, 4, 4161-4171.

24. Z. C. Yang, X. Li and J. Wang, Carbon, 2011, 49, 5207-5212.

25. Y. Song, S. Zhu, S. Xiang, X. Zhao, J. Zhang, H. Zhang, Y. Fu and B. Yang, Nanoscale, 2014, 6, 4676-4682.

26. A. Zhao, C. Zhao, M. Li, J. Ren and X. Qu, Anal. Chim. Acta, 2014, 809, 128-133.

27. K. Qu, J. Wang, J. Ren and X. Qu, Chem-Eur. J., 2013, 19, 7243-7249.

28. S. Zhu, Q. Meng, L. Wang, J. Zhang, Y. Song, H. Jin, K. Zhang, H. Sun, H. Wang and B. Yang, Angew. Chem., 2013, 125, 4045-4049.

29. X. Sun, X. Ma, C. V. Kumar and Y. Lei, Anal. Methods-UK, 2014, 6, 8464-8468

Publisher's Note Engineered Science Publisher remains neutral with regard to jurisdictional claims in published maps and institutional affiliations. 